\title{
Learning Dashboards: an Overview and Future Research Opportunities
}

\author{
Katrien Verbert ${ }^{1,2}$, Sten Govaerts ${ }^{1,3}$, Erik Duval ${ }^{1}$, Jose Luis Santos ${ }^{1}$, Frans Van Assche ${ }^{1}$, Gonzalo \\ Parra $^{1}$, Joris Klerkx ${ }^{1}$ \\ ${ }^{I}$ Department of Computer Science - KU Leuven \\ \{erik.duval, joseluis.santos, frans.vanassche, gonzalo.parra, joris.klerkx\}@cs.kuleuven.be \\ ${ }^{2}$ Department of Computer Science - Eindhoven University of Technology \\ k.verbert@tue.nl \\ ${ }^{3}$ Departement of Computer Science - EPFL \\ sten.govaerts@epfl.ch \\ Corresponding author: \\ Erik Duval \\ erik.duval@cs.kuleuven.be \\ Phone: +32-16-32.70.66 \\ Celestijnenlaan 200A - bus 2402 \\ B-3001 Leuven, Belgium
}

\section{Abstract}

In this paper, we present work on learning analytics that aims to support learners and teachers through dashboard applications, ranging from small mobile applications to learnscapes on large public displays. Dashboards typically capture and visualize traces of learning activities, in order to promote awareness, reflection and sense-making, and to enable learners to define goals and track progress towards these goals. Based on an analysis of our own work and a broad range of similar learning dashboards, we identify HCI issues for this exciting research area.

Keywords: learning analytics, information visualization, dashboards, HCI

\section{Introduction}

There is a growing movement to more open learning environments. For instance, Personal Learning Environments replace monolithic Learning Management Systems with user configurable sets of widgets [1]. Learning infrastructures provide generic services for learning, for instance through registries ${ }^{1}$, or open educational resource infrastructures [2].

At the same time, there is a growing movement of quantified self in medicine [3], sports, many other fields and, indeed, learning [4]. The basic idea in many of these initiatives is to enable users to track their activities, in order to enable self-analysis and comparison with other users, often by

\footnotetext{
${ }^{1}$ http://www.learningregistry.org/
} 
aggregating traces into metrics (weight lost, or total distance covered), or by visualizing these activity traces.

More specifically, the field of learning analytics focuses on tracking learning activities, and the context in which these activities occur, to promote awareness and reflection through algorithmic analysis (in educational data mining [5]) or information visualization.

We have designed, developed and evaluated a suite of tools for tracking learning activities and visualizing them as learning dashboards over the full gamma from mobile devices over tablet and laptop to desktop computers, up to tabletops and large public displays. We have carried out these developments and evaluations in projects with real life test beds - often using a participatory design approach. As an example, Fig. 1 illustrates a mobile client: the learner dashboard shows the number of relevant course tweets, links to other relevant information, and a progress indicator that takes into account the time investment of the student, progress made in the course, the course schedule, etc. Such mobile clients provide exciting affordances for automatic tracking of learning activities - for instance, students can track time spent, proximity, etc. or 'check in' for a lecture in a foursquare ${ }^{2}$ type of way.

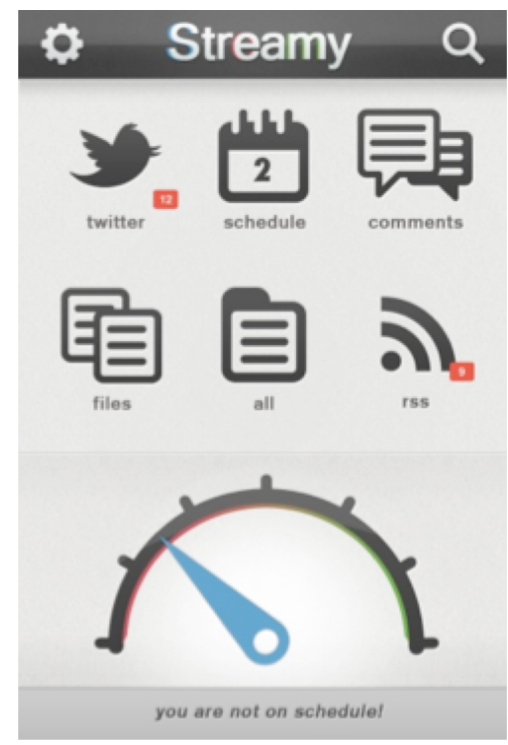

Fig. 1 a mobile dashboard designed and developed by our students

On laptop and desktop environments, we have developed numerous trackers for learning activities (leveraging existing tools such as wakoopa ${ }^{3}$, rescuetime ${ }^{4}$ and the rabbit Eclipse plugin ${ }^{5}$ ). We have

\footnotetext{
${ }^{2}$ https://foursquare.com/

${ }^{3} \mathrm{http://wakoopa.com/}$

${ }^{4}$ https://www.rescuetime.com/

${ }^{5}$ http://code.google.com/p/rabbit-eclipse/
} 
made these data available in visualizations that rely on OpenSocial widgets [6], so that learners and teachers can compose their own dashboard.

In this paper, we analyze a wide range of learning dashboard applications that have been deployed and evaluated in recent years. Similar to our work, these dashboards capture data about learner activities and visualize these data to support awareness, reflection, sense-making and impact, for instance by having an influence on behavior change - as illustrated in Fig. 2. Impact can also include new meaning or insights gained from these visualizations.

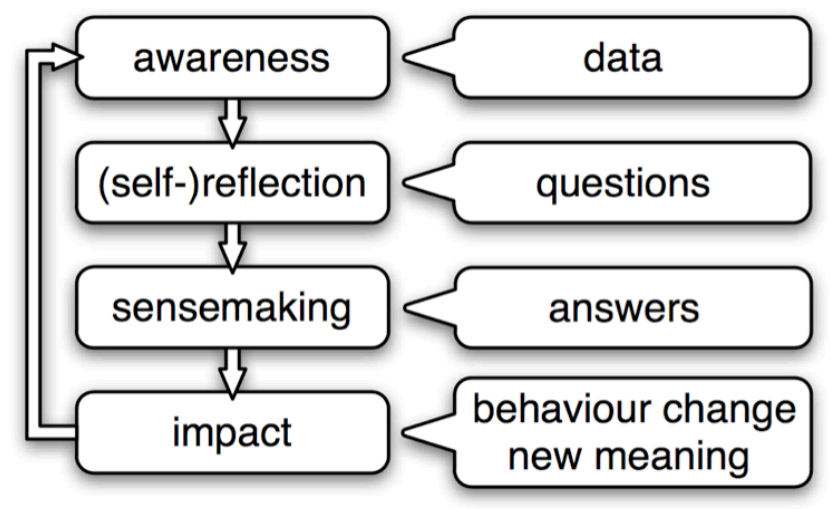

Fig. 2 learning analytics process model [7]

The research contribution of this paper is twofold: first, we present an analysis of the state of the art in this emerging research area. We focus specifically on the following research questions:

- RQ1: What are relevant user actions?

- $\quad$ RQ2: How can data on relevant actions be captured?

- RQ3: How are awareness \& self-reflection enabled for different kinds of users through appropriate devices?

- RQ4: How can learning analytics dashboard applications be evaluated? How can the impact of these visualizations on user behavior be measured?

Second, based on an analysis of existing learning dashboard applications, we outline opportunities for future research in this area.

This paper is organized as follows: the next section presents an overview of learning analytics dashboard applications. Then, we discuss which actions are considered relevant (RQ1), which sensors are used to capture these data (RQ2), for which users visualizations of these data are provided on appropriate devices and for which purposes (RQ3) and how existing dashboards have been evaluated to assess their impact on learning or teaching (RQ4). Future research challenges are presented next.. Conclusions and take away messages from this analysis are presented in the last section. 


\section{Overview of learning analytics dashboards}

Learning dashboards that have been deployed in recent years can be broadly categorized in three groups: 1) dashboards that support traditional face-to-face lectures, 2) dashboards that support face-to-face group work and 3) dashboards that support awareness, reflection, sense-making and behavior change in online or blended learning. We present some prominent examples in this section. This list is by no means exhaustive, but it presents nevertheless a broad range of interesting work in these areas.

We present the dashboards in the categories in which they have been deployed (i.e. traditional face-to-face lectures, face-to-face group work or blended/online learning settings) - but many dashboards may be applicable in other categories as well. We analyze the commonalities and differences of these dashboards in the next section.

\section{Dashboards to support traditional face-to-face lectures}

Several dashboards have been presented to support traditional face-to-face lectures. The general objective of these dashboards is often to support the teacher in receiving live feedback from students during large lectures in order to adapt her teaching. As an example in this category, Yu et al. [8] present a dashboard that supports live awareness for a teacher on learner agreement or disagreement while she is teaching. The system detects learner attitudes by tracking head nods or shakes and voice with a built in camera and microphone of a desktop PC.

Other dashboards are used to engage students during lecture sessions. Backstage [9] is an interesting example that visualizes twitter activity of students during a face-to-face lecture. Students can compare their twitter activity with that of their peers. As illustrated in Fig. 3, a student can compare her activity to the average activity and to activities of other individual students who are attending the lecture. The color of each disc, that represents a student or the average, presents positive ratings received on tweets (from green over yellow to red in decreasing order).

Classroom salon [10] is a dashboard that visualizes social collaboration. The dashboard allows teachers to create, manage, and analyze social networks (called Salons). Students in a Salon can cooperatively create, comment on, and modify documents. The dashboard visualizes how much each member contributed, percentages of responses categorized by tag and how many responses individual members made to questions. This dashboard has been used in settings where students collaboratively annotate lecture slides during a face-to-face lecture. Similarly, Slice 2.0 [11] is a framework that interconnects tablets of students with slides used by the teacher. A dashboard 
application allows the teacher to monitor students and visualizes current notes of students on slides and questions. The teacher can also interact directly with these notes and display notes of a particular student for group discussion on a large display.

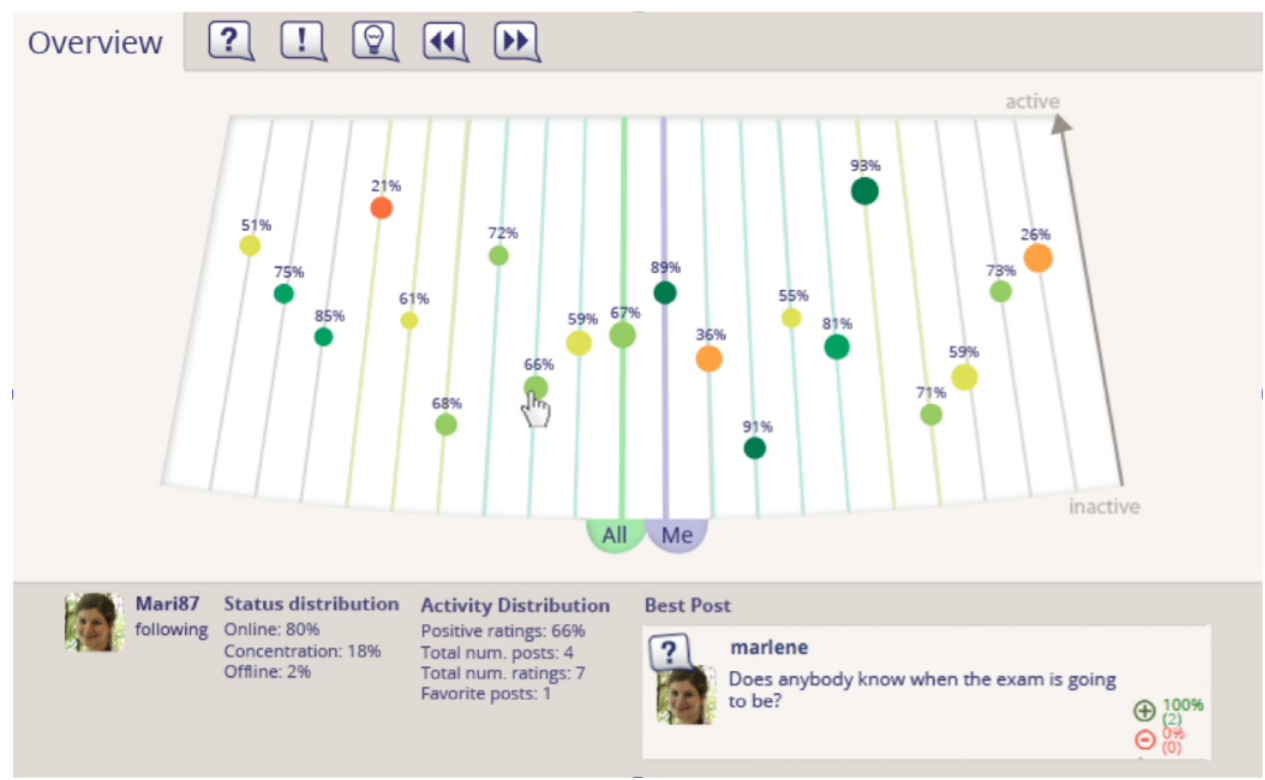

Fig. 3 Backstage [9]

\section{Dashboards to support face-to-face group work and classroom orchestration}

Other dashboards focus on classroom orchestration and support for teachers to manage group work. Typically, such orchestration refers to the real-time classroom management of multiple activities [12]. TinkerBoard [13] is an example that visualizes which activity each group is doing (building models, doing quizzes, running simulations, etc.) and how intensively. The dashboard is part of TinkerLamp 2.0, a system that tracks collaboration on tabletops. Activity information is visualized on a large display and used to support awareness and reflection in a classroom.

Collaid (Collaborative Learning Aid) [14] captures learner collaboration data on tabletops and visualizes this data to give a teacher insight in collaboration among students. The system uses a tabletop, a microphone array and a depth sensor to capture audio, physical and positioning traces of activity of each tabletop user. This data is then visualized on a tablet for the teacher as a visual interactive dashboard (see Fig. 4). Each colored circle represents a learner. The first radar chart shows a blue and red triangle that depicts the verbal and physical participation in the group. The closer the corner of the triangle is to the circle, the more that student was participating. The second radar chart measures the quantity and symmetry of physical actions on the tabletop. The third chart summarizes the level of collaboration detected by the system. 


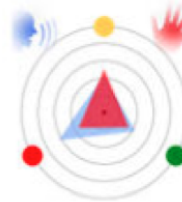

1

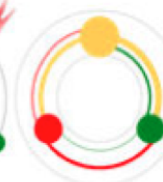

2

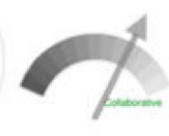

3

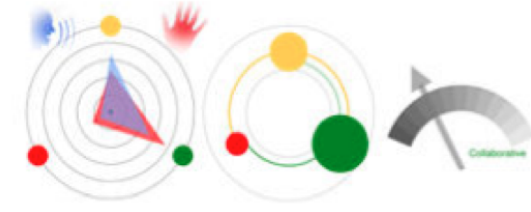

2

3

Fig. 4 Overview visualizations of Collaid [14]. Left: a balanced group; Right: a group in which one member (red circles) was completely disengaged from the activity

Similarly, Class-on $[15,16]$ visualizes learning activities on a tablet, in order to provide awareness for teachers. More specifically, the system keeps track of help requests by students during lab sessions. In addition, progress of students is tracked and the dashboard visualizes this information on a tablet used by the teacher, so that she can decide which group to help next during a face-toface session.

Morris et al. [17] present a tabletop application that includes interactive visualizations of collaboration between students. Histograms appear on the table in the region directly in front of each user, and reflect the number of answers contributed by that group member based on touch interactions with the table. Contrary to the previous examples, this application visualizes activity indicators on the tabletop rather than on a different device.

\section{Dashboards to support blended or online learning settings}

A number of dashboards have been developed to support students and teachers during blended or online learning. Course signals [18] is a dashboard that predicts and visualizes learning outcomes based on three data sources: grades in the course so far, time on task and past performance. If grades in the course so far are below a certain threshold specified by the teacher, a student will see a red color signal for the course. When they are above the threshold, past performance in other courses and time on task are used to calculate whether a student is on track (green light) or whether she may need to improve her activities for the course (orange light). A similar dashboard has been developed at Carnegie Mellon University [19]. This dashboard is presented in Fig. 5 and presents with similar colors the extent to which a student is achieving her goals for the course. In contrast to Course Signals, this dashboard presents at a more fine grained level which concepts may need additional attention of the student and how she is performing on different course activities. These indicators are among others calculated based on self-assessments activities that are interweaved in online course material. 


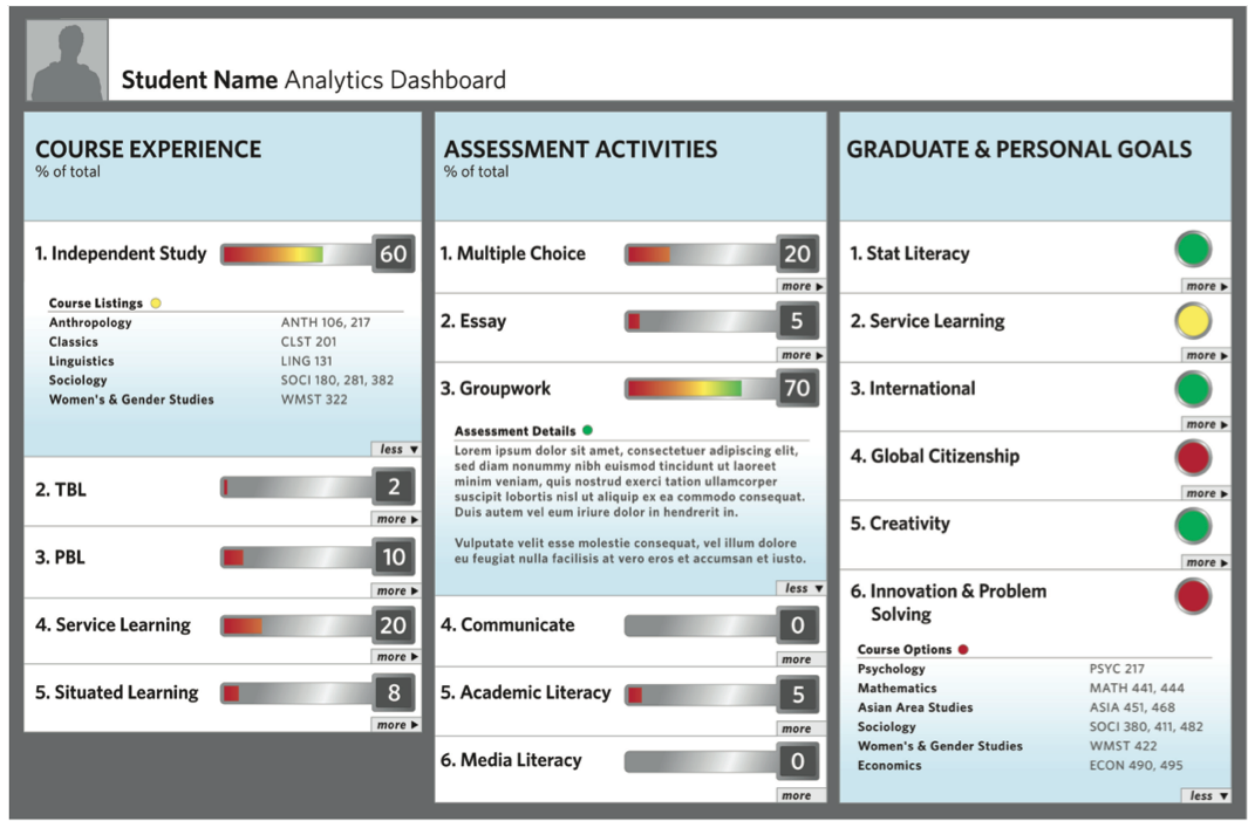

Fig. 5 OLI dashboard [19]

Student Activity Meter (SAM) [20] - one of our visualization tools - provides visualizations of progress in the course for teachers and learners. Fig. 6 illustrates time spending of students (grey lines) over the course period. The statistics panel on the right provides further details - such as minimum, maximum and average activity. SAM contains different configurable visualizations of time spent and resource use tracked from various learning environments - including a traditional learning management system such as Moodle. LOCO-Analyst [21], Moodle dashboard [22] and GLASS [23] are similar dashboards that provide teachers with feedback on activities and performance of students. Typically, time spent and artefacts produced are visualized to give a teacher insight into efforts of students. In addition, some of these dashboards visualize results of self-assessments to give an indication of learning progress. Similarly, Student Inspector [24] visualizes usage data of the ActiveMath (http://www.activemath.org/) learning environment to provide details about performance based on test scores, typical learner errors and the strong and weak topics of students, as identified by self-assessments. Tell Me More [25] also provides visualizations to support awareness based on results of exercises. Finally, CALMSystem [26] visualizes knowledge levels as detected by the system through self-assessments in comparison to how a student perceives her current knowledge on different topics. This data is visualized to support awareness and reflection. The overall goal is to improve self-assessment of students. 


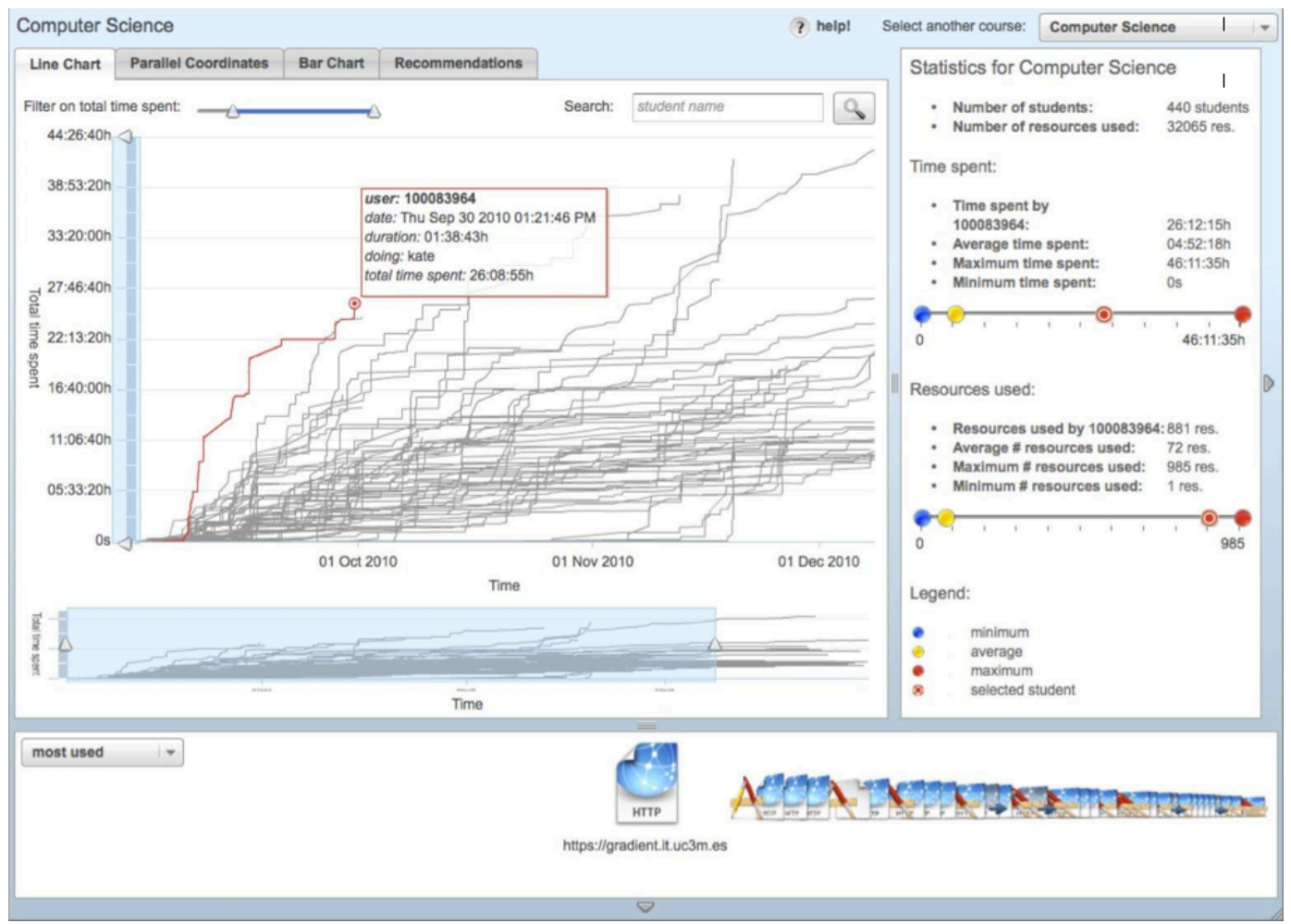

Fig. 6 The Student Activity Meter (SAM)

Other dashboards also incorporate a learning schedule to support awareness. Chen et al. [27] present a dashboard that supports learning status awareness (i.e. if a learner is available and online), schedule awareness that visualizes assignments of teachers, and learning support awareness. The latter suggests potential peer learners for a learning activity. Data to provide such awareness is constructed from self-assessments, learning behavior within the learning management system, teacher assignments and schedule, and test results of the students. Notifications are sent to learners by SMS - after approval of a teacher. Teacher Advisor (TADV) [28] relies on similar manual interventions of a teacher to propagate automatically generated advice to a learner.

The use of a mobile dashboard is also evaluated as part of StepUp! [6] - another example of our dashboards that visualizes social interaction, time spent, artefacts produced and resource use. Fig. 7 presents this mobile version that shows how much progress a student has made towards specific activities of the course - such as posting comments on blogs of peer students and tweeting current activities. 


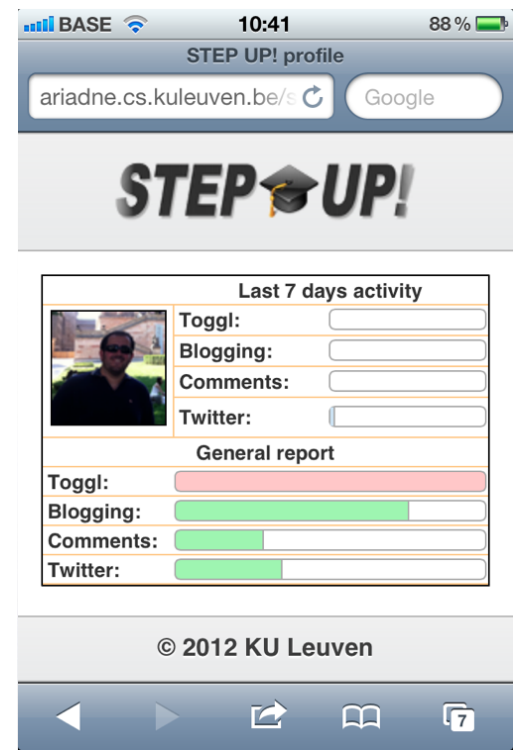

Fig. 7 mobile dashboard StepUp! [29]

iTree [30] is an early example of a mobile dashboard that is used to engage students with the use of a class forum. As illustrated in Fig. 8, the dashboard visualizes contributions of students by a tree. The growth of the tree is affected by four variables: (1) number of posts, (2) the number of times posts of a student are read, (3) the number of replies to posts, and (4) ratio of total forum posts to replies.

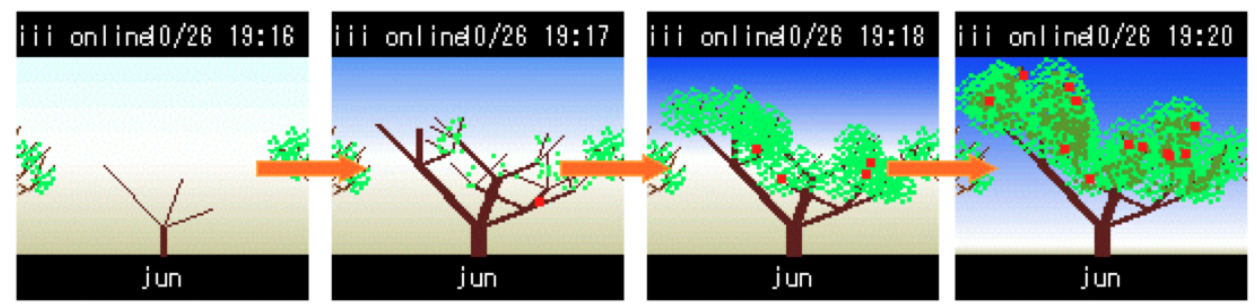

Fig. 8 iTree [30]

Visualization of such social interactions and online collaboration is also the key focus of SNAPP [31], TUT Circle Dashboard [32] and Classroom view [33]. SNAPP [31] visualizes the evolution of social interactions among learners in online forums. The dashboard provides real-time data visualizations, social network metrics and event annotation tools to enable teachers to intervene. TUT Circle Dashboard [32] presents activities of the learners and peers within the learner's network. The authors present an evaluation of motivational aspects of learners with the use of the dashboard. Similar to SNAPP, Classroom View [33] is developed to support awareness for teachers. The dashboard visualizes collaboration and current activity in online group work. 


\section{Analysis of learning analytics dashboards}

Table 1 presents an overview of several characteristics of learning analytics dashboard applications that we presented in the previous section. We analyze these characteristics along the lines of the research questions presented in the introduction.

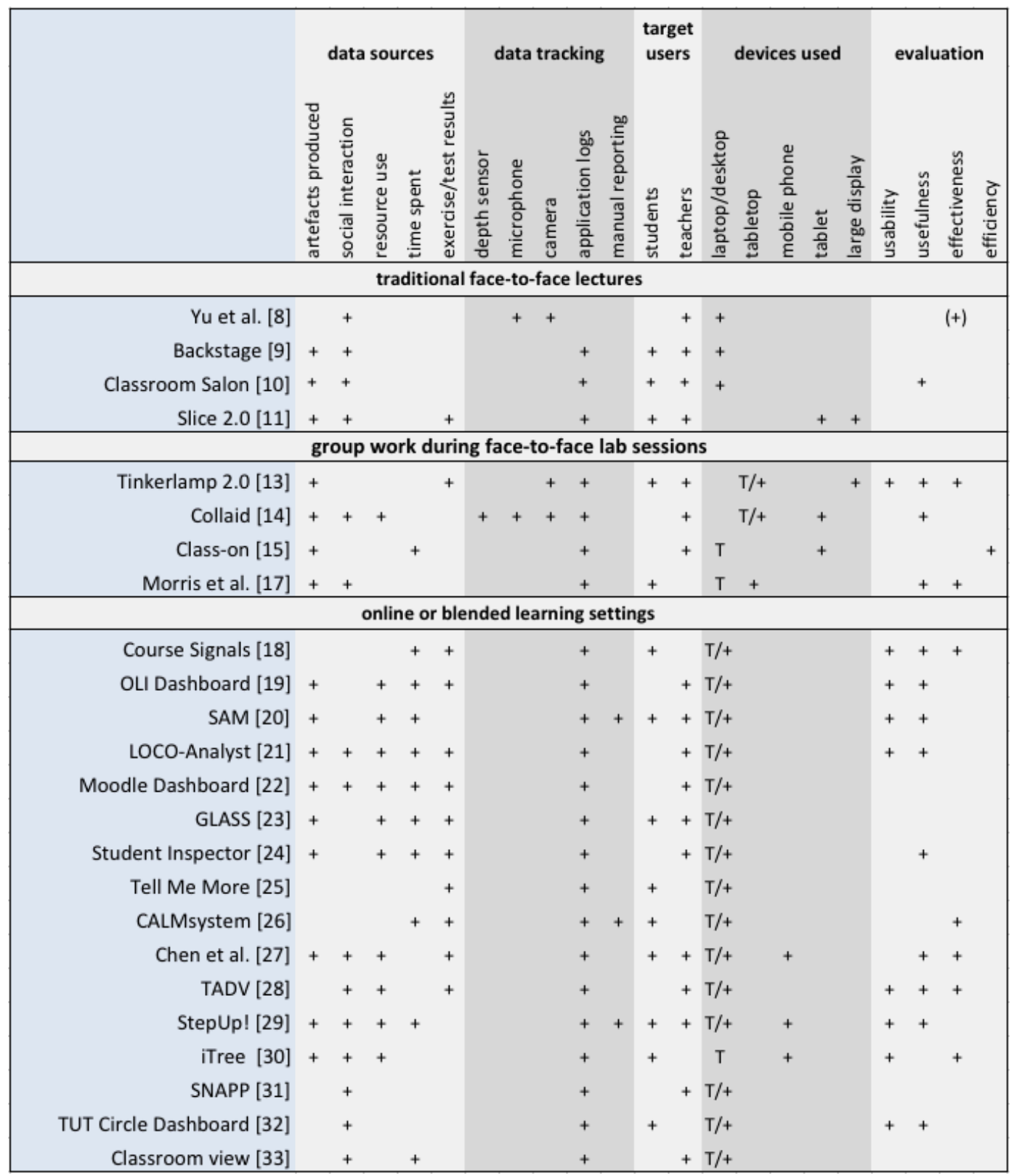

Table 1 learning analytics dashboard applications

\section{RQ1: What are relevant user actions?}

This section makes explicit what researchers and developers of existing tool are tracking and thus probably believe is relevant. 
As presented in the first column of Table 1, 16 out of 24 dashboards visualize artefacts produced by learners. Artifacts include posts on blogs [29], fora [20-22, 30] or Twitter [9, 29], responses to questions $[11,17,19,20]$, help requests [15], annotations [11, 10] or, more generally, resources students created [10, 13, 19-24, 27]. Such resources can be documents that are shared [10, 19, 27] or very specific artifacts like layouts on tabletops $[13,14,17]$ that enable students to compare different layouts they have built over time.

Social interaction is a second commonly tracked and visualized element. Examples include analysis of amount of speech by the learner in face-to-face group work [14] or interactions and ratings of tweets [9, 29], comments on blog [29] and forum posts [21, 30-32], or chat messages between learners [21]. Yu et al. [8] present some interesting work on measuring social signals from students in terms of politeness, stress, agreement and disagreement.

Resource use is tracked by 11 systems. iTree measures reads of forum posts [30] as a basis to estimate engagement. Most other dashboards track resource use as a basis to provide awareness for teachers on resource use by their students - either to identify indicators on the relevancy of resources or to estimate effort. While not used by the dashboards we surveyed, resource use has been used in examples of adaptive hypermedia and recommender systems to estimate knowledge levels of the learner as well [36].

Time spent is also included in 11 dashboards. SAM [20], Student Inspector [24], LOCO-Analyst [21], Moodle dashboard [22] and StepUp! [29] visualize time spent so that teachers can identify potential students at risk. In addition, time spent is sometimes visualized for students, so that they can compare their efforts with those of their peers [20, 23, 29]. Class-on [15] tracks time spent by teachers, in order to provide feedback to a teacher on whom to help next during lab sessions. Other dashboards such as Course Signals [18] and CALMSystem [26] use time as a basis to estimate the knowledge level of a learner on a specific concept.

Test and self-assessment results that capture knowledge levels are used by 12 out of 24 dashboards that are used in blended or online learning settings. Such data are used to get a better indication of learning progress than for instance time spent provides. In Slice 2.0 [11] and Tinkerlamp 2.0 [13], responses of students on questions during a face-to-face session are visualized to support reflection and group discussion.

\section{RQ2: How can data on relevant actions be captured?}

Data on relevant actions are captured by physical sensors, such as cameras and microphones, virtual sensors such as application logs and through manual reporting by learners or teachers. 
- Physical sensors: Collaid [14] uses a microphone array and a depth sensor to capture audio and positioning traces of activity. This information is used to identify which student contributed which artefacts to group work. Yu et al. [8] analyze video and audio during face-to-face sessions to detect emotional responses of learners.

- Almost all systems rely on virtual sensors that track actions within a software application. Such actions include posting, reading or replying on a student forum in iTree [30] and SNAPP [31]. Chen et al. [27] capture logon and logoff actions and updates by students on different learning modules. Each of the activities has timestamp and other properties such as message content, identity information of the contributor and a category. Such information is also tracked by other systems, including Classroom Salon [10], to visualize how much each learner contributed and percentages of responses categorized by tag.

- StepUp! [29] and SAM [20] have been used in setups where manual tracking tools are used to capture time spent - such as Toggl ${ }^{6}$. In CALMystem [26], users are asked to provide self-assessment ratings for topics. This data is used to compare automatically generated indicators of knowledge levels with manual assessments as a basis to support reflection.

RQ3: How are awareness, reflection, sense-making and impact enabled for different kinds of users through appropriate devices?

The fourth column of Table 1 presents the devices that are used by learning analytics dashboard applications. We distinguish between devices that are used to track interactions of learners, for instance through application logs (indicated by ' $\mathrm{T}$ ' in Table 1), and devices that are used to visualize this data for teachers or students (indicated by '+'). Desktops and laptops are the most used devices to both visualize and track data.

Tablets are increasingly used in recent examples of learning analytics dashboard applications - for instance in Slice 2.0 [11] and Class-on [15]. Slice 2.0 enables students to interact with lecture slides during face-to-face sessions. Class-on visualizes activities on a tablet to provide awareness for teachers during lab sessions. Mobile devices are used in some examples, for instance by Chen et al. [27], iTree [30] and StepUp! [29]. Benefits include increased mobility and ubiquitous access to visualizations and statistics.

Tabletops are becoming more popular to facilitate group work. In most applications, tabletops are used to track interactions on the tabletop - for instance in Collaid [14] and Tinkerlamp 2.0 [13]. This data is then visualized on a different device, for instance on a tablet, and used to increase awareness for teachers. Moris et al. [17] present a dashboard that visualizes collaboration indicators on a tabletop to provide awareness for students.

\footnotetext{
${ }^{6}$ https://www.toggl.com/
} 
RQ4: How are learning analytics dashboard applications evaluated? How can impact of dashboards on learning behavior be measured?

Effectiveness, efficiency, usefulness and usability of learning dashboard applications have been evaluated in several ways.

Effectiveness has been evaluated for seven out of 24 dashboards and has in most cases been evaluated in controlled settings - i.e. during a single session with an experimental group using the dashboard and a control group without such support. Course Signals [18] is the only dashboard that has been evaluated in a large scale study over three academic years. Effectiveness has been measured in terms of better engagement $[17,30]$, higher grades $[28,18,27]$ or post-test results [13], lower retention rates [18] and improved self-assessment [26]. Results of a long-term experiment with Course Signal [18] indicate that there is an impact on retention rates and grades.. A significant difference was found in improving self-assessment in an evaluation of CALMSystem [26]. In other evaluations, grades or post-test results were higher in 2 of 3 controlled case studies although no significance results are presented in [27]. Although no difference in grades was found in an evaluation of TADV [28], the overall satisfaction with the course for learners using the dashboard was higher. Among others, this satisfaction was measured in terms of enjoyment, selfesteem, contact with facilitators and recommending the course to other students. In other experiments, engagement of the students is measured to get an indication of potential impact. Results of Morris et al. [17] indicate that there was no increase of engagement when students used the dashboard. Results of iTree [30] indicate that the dashboard does not encourage learners to post messages on a forum, but there is an increase in reading of posts.

Efficiency was measured in an evaluation experiment of Class-on [16] and assessed whether the use of a dashboard during classroom sessions helps to distribute time of a teacher in a more fair way. Although still preliminary, some trends in the data are shown that indicate improved efficiency during face-to-face group work.

Usability and usefulness evaluations have been conducted most often - either with teachers or students, or both. These evaluations often focus on asking teachers questions related to finding learners at risk or asking learners how well they think they are performing in a course. In addition, questionnaires were used to gain an indication of perceived usefulness for improving learning and teaching. Perceived usefulness of Student Inspector [24] and LOCO-Analyst [21] was for instance evaluated with teachers and was high for both dashboards. Results of our evaluations with SAM and StepUp! indicate that perceived usefulness is often higher for teachers than for students [20]. In addition, we have measured and compared usefulness with different kinds of tracked data in seven case studies. Results indicate that students perceive usefulness higher if dashboards present a more complete description of their learning activities. For instance, in case studies where we 
visualized social interaction, perceived usefulness was lower than in case studies where we also tracked time spent. Evaluation results of LOCO-Analyst [21] also indicate that perceived usefulness was significantly higher in a case study where more data visualizations were used to provide insight in learning activity.

\section{Research issues}

We briefly discuss some of the most important research issues below and our ideas to address these issues in future research experiments. This section is organized by the same research questions mentioned above.

\section{RQ1: What are relevant user actions?}

In learning contexts, there seems to be limited consensus on what are relevant data... Time of day, location, whom the learner is with or what device she is using, what the teacher has had for lunch, the background noise: maybe all of these are important - and maybe not. As mobile devices proliferate, and as these devices integrate more sensors, there are more and more characteristics that we can measure. Still, it is not because we can measure them that they are relevant. However, it is often difficult to figure out beforehand what will be relevant, why or how...

Results of our analysis in the previous section indicate that artefacts produced, time spent, social interaction, resource use and exercise and test results are commonly used by learning analytics dashboard applications. These data have been captured by several dashboard applications to support awareness, reflection and sense-making for teachers and for students. Artefacts produced and social interaction are most often used by these dashboards. Both dimensions are used in $66 \%$ of the dashboards that we surveyed. Time spent, resource use and exercise and test results are used by approximately $50 \%$ of the surveyed dashboards.

In some of our own evaluation studies [20,29], we have tried to assess to which extent these data have an influence on the usefulness of dashboards. In these case studies, we have deployed dashboards with different data sources, including time spent, resource use and social interaction. Results of seven case studies are summarized in Fig. 9. The first set of boxplots of Fig. 9 summarizes answers to evaluation questions regarding perceived usefulness of social interaction in seven case studies, with 59 learners and 41 teachers. The second and third set present perceived usefulness of resource use and time spent. The last set of boxplot presents overall perceived usefulness of learning dashboards. The bottom rows of boxplots summarize the results of all studies for teachers and students.

From our evaluation studies, it is clear that learners consider social interaction only somewhat useful - maybe also because most of our courses take place in a blended setting, where learners 
often meet face-to-face. Teachers consider visualization of social interaction more important. Social interaction visualization is for instance considered useful to identify students who do not collaborate with others or students who excel at this.

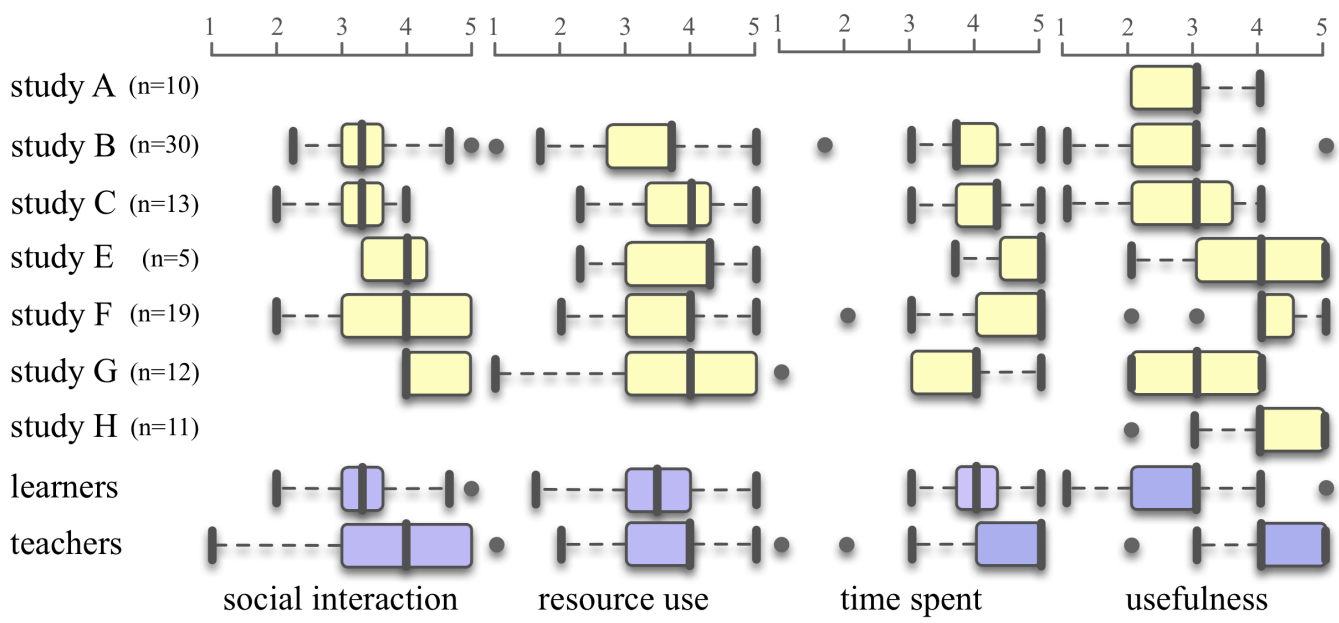

Fig. 9 what data to track and perceived usefulness ( $1=$ very low, $5=$ very high $)$

Learners make use of resources such as learning objects, Open Educational Resources, documents and web resources. Teachers are also interested in such data. They want to know how often and when students use resources they provide, as well as which external resources learners collect themselves. Learners seem to be less interested in these data as such, though they do value for instance recommendations that are based on these data [34].

Teachers find learner effort very important and perceive the support that learning dashboards provide in this area as very high. (We mainly measured time spent as an indicator for effort.) Learners also have a positive perception, though less outspoken than teachers.

Overall perceived usefulness is different for students than for teachers. Students often worry that collected data traces do not represent all the work done, whereas teachers do perceive (partial) data useful as indicators to gain insight in student activity and to find potential students at risk. In addition, the setup of the course may influence usefulness perception. For instance, we obtained better results with students working in groups and on a common topic, than with students working individually [35]. Thus, the relevancy of the actions can also be influenced by many contextual variables [36].

How these data interrelate, which one is more important, etc. are open research questions. Moreover, all of the data mentioned above relate to quantitative data. One area of research is to investigate whether we can augment this approach with qualitative data: for instance, the number of re-tweets or comments on a blog post can indicate how relevant a communication is. Likewise, 
we could ask students to rate artefacts produced by their peers in an explicit way, through a simple star based system, or a thumbs up - thumbs down facility, or a more sophisticated rubric that can become more of a peer review approach for networked learning. The idea has already been explored by the authors of the Backstage dashboard [9]. We elaborate in section RQ4 on our own work in this area.

More generally, there is a need to research what data about the learning process and the learner can be useful for learning analytics dashboard applications. Maybe some mouse clicks or physical interactions are not related to the learning activity (for instance: quick email or chat interrupt, or leaving the room to get a coffee), but then again, maybe they are and it is often difficult to figure out what activity is relevant at which point in time. Possibly, sometimes it can be useful to apply a more bottom-up approach, i.e. to track because it is possible, even if we do not know yet how we will use the data. Doing so may help us to discover relevancy that we are as yet unaware of, for instance with respect to when we learn, or where, or with whom, etc. What exactly is relevant and for what purpose remains quite unclear and needs further research.

\section{RQ2: How can data on relevant actions be captured?}

Most existing learning analytics dashboards rely on virtual sensors that track interactions of learners with tools and resources. In our studies, we often rely on trackers for laptop or desktop interactions, social media for learner interactions (through twitter hash tags or blog comments, for instance). In contrast to almost all other dashboards surveyed in this article, we have also applied manual reporting by learners. Such manual reporting, supported using twitter or Toggl in our case studies, may be useful as it can serve as a 'reflection trigger' that will help learners to reflect on what they are doing and thus potentially increase their effectiveness and efficiency [37]. On the other hand, automatic tracking is more consistent, and systematic manual reporting requires considerable discipline.

In the case of automated tracking, one major concern is to track all, or at least a major part of learning activities. Indeed, we know from our evaluation studies that learners rate usefulness of learning dashboards low when many relevant activities happened outside the tracked learning environment. Comprehensive tracking is difficult in more closed LMS environments, as they typically cover only the 'tip of the iceberg' [38]. It is also difficult in more open Personal Learning Environments, as these can include a wide variety of tools and services, though we did receive positive results with aggregating learning traces from diverse tools like twitter, blog posts and comments, software development environments, etc.

Only a few examples already incorporate the use of physical sensors such as cameras or microphones to capture learner actions. The use of these sensors to monitor and advice on face-to- 
face group work has been researched in the CSCL (Computer Supported Collaborative Learning) research area [39], but has only to a limited extent been incorporated in learning dashboard applications that provide real-time feedback to learners or teachers. The use of these sensors is also determined by privacy constraints of the users. For instance, our students sometimes accept to be tracked during lab sessions [29], but they often do not want to be tracked when they work outside the lab.

The use of additional sensors to capture learner actions and physiological responses is of particular interest as well. For instance, Bakker et al. [40] recently presented research on the use of sensors to capture physiological responses as a basis to estimate stress levels and to provide feedback to employees on their current work schedule. Extending such research to provide awareness and reflection in learning settings and the impact of such awareness is an exciting research topic. The interest in this area is reflected by a number of contributions to the LAK (Learning Analytics and Knowledge) conference [41, 42] and MATEL (Motivational and Affective Aspects of TEL) workshop series [43, 44].

\section{RQ3: How are awareness, reflection, sense-making and impact enabled for different kinds of users through appropriate devices?}

The majority of the surveyed dashboards visualize data for use on laptops or desktops. Only a few examples visualize data on tablets for use in classroom [14][15]. Also interesting is that so far only one dashboard visualizes data on tabletops [17]. Although some prominent research has been done to track data from tabletops, the use of large displays as a means to provide teachers or students with insights into behavior patterns is limited. Also mobile devices are not yet used by many learning analytics dashboard applications. In one of our case studies, we deployed a mobile version of StepUp! [29] to better engage our students with the use of learning dashboards.

In summary, there is limited use so far of the affordances that both mobile devices and larger displays provide for tracking and visualizing data, respectively.

Some of the central future research directions are listed below:

How can we exploit novel opportunities in mobile devices for supporting communication and collaboration between learners and with teachers, which is especially relevant in a Computer Supported Collaborative Learning (CSCL) setting [39], the more so as these devices can capture context information?

How can we design physical spaces that promote learning rather than hinder it, especially in the case of tabletops and large public displays, where the impact of the physical environment on the 
user experience is sometimes higher and, vice versa, the devices have a higher impact on the physical setting [45]?

What kind of data and service infrastructure can best support the applications we envision? Of particular relevance here is a linked open data approach that can integrate well with the Web infrastructure [46] and that can support an open analytics infrastructure [47]?

How can we enhance and exploit facilities for seamless transition from mobile over tablet and laptop to desktop, tabletop and large public displays. Issues here include coherence, synchronization, screen sharing, device shifting, complementarity and simultaneity (see http://precious-forever.com/)?

\section{RQ4: How are learning analytics dashboard applications evaluated? How can impact of dashboards on learning behavior be measured?}

Whereas usability and usefulness are relatively easy to evaluate (and we have done many such evaluations of our tools), gains in efficiency and effectiveness, for instance in the form of learning impact, is much harder to evaluate, as this requires longer-term and larger-scale evaluations. Evaluations that have been conducted so far are often conducted in a controlled setting with an experimental and a control group. Whereas these experiments are interesting, it is difficult to generalize the results of these studies. In addition, evaluation results are often contradictory - in some setups researchers were able to identify a significant increase of grades, whereas in other experiments no significant difference in test scores was found.

Longitudinal studies such as [18] rely impact on correlations of the tool use with some positive learning effects such as retention rates or improvement in grades. Other evaluations focus on better engagement $[17,30]$ or improvement of self-assessment [26].

As illustrated in Table 1, most evaluations of dashboards are limited to usability and perceived usefulness. These evaluations often rely on small-scale user studies with teachers or students and some example tasks they should perform by using dashboards. Although these studies are interesting, they provide little evidence whether learning analytics dashboards are solving real issues and needs of students or teachers. As pointed out by Baillie et al. [48] and Killen et al. [49], there are differences between students and teachers about the perception of learning issues - and therefore the goals and issues that should be supported by learning dashboards.

In our own work, we have tried to address this issue by asking students about their actual needs and issues [35]. Through brainstorming sessions with our students, we identified and prioritized learning issues and needs. In a second step, we deployed StepUp! during one month and we 
evaluated to which extent our dashboard addresses the issues and needs identified earlier in different courses. We strongly believe that similar critical evaluations of the actual use of learning analytics dashboards are required to gain insight into the added value of this research area. Student perceptions of added value are not the only criterion, but certainly an important one. Asking students about their actual issues can help to better understand their needs, and is key to better target dashboards towards improvements in effectiveness of learning.

How we can enable goal setting and connect it with the visualizations, so as to close the feedback loop and enable learners and teachers to react to what they observe and then track the effect of their reactions, is another interesting research question. We are experimenting with playful gamification approaches that present their own challenges [29], for instance around trivialization and control.

Positive attitude towards gamified applications is influenced by social influence and perceived recognition [50]. Mozilla Open Badges rely on these factors to build a platform that enable users to share their achievements through social networks [51]. However, some experiments reported that whilst students with a gamified approach scored better in practical assignments, they participated less and performed poorly in written examinations [52].

Our experience with such an approach is positive so far. We have deployed a badge reward system with badges that have positive and negative connotations [53]. Students who are actively commenting on other students' blogs receive a positive badge, whereas students who did not comment on any blog receive a negative badge. Our experience so far is that badges motivate our students to be more active during the course and help them to be aware what goals they have to reach. Such a better engagement is a key requirement to facilitate evaluation studies that assess impact of dashboards over a longer period of time. Further research needs to be conducted in order to demonstrate that badges can trigger reflection and help students to drive conclusions out of their own and peers' activity.

\section{Conclusion}

In this article, we have presented an analysis of learning dashboards that have been deployed to support awareness, reflection, sense-making and impact on learning. The research contributions of this article are twofold. First, an overview of existing learning analytics dashboards applications has been presented and the affordances that these applications provide to support learning or teaching. Second, we have outlined several research issues for the development and evaluation of dashboards for learning based on this analysis. 
Results indicate that there has been much advancement on the development of learning dashboards in recent years. Many promising prototypes illustrate the potential and opportunities that these applications create.

Nevertheless, important challenges related to capturing of data traces, and deployment and evaluation of dashboards remain that need to be tackled in order to gain insight into actual impact. From current mixed and limited evaluation results, it is clear that learning analytics research is still in its infancy and that current tools and applications present a mixed picture. Take away messages of this analysis include:

1 Future research is needed to assess the usefulness of different data types for learning analytics dashboards applications. So far, researchers have mainly tracked resource use, time spent, social interaction, artefacts produced and exercise and test results. Little research has been done to assess the impact of these data on the usefulness of dashboards. In addition, there are many additional data sources that may be relevant to increase awareness and reflection about the learning process. Recent progress in emotion and stress analytics from physiological data [40] is just one of the exciting new research opportunities to capture a much richer set of relevant data.

2 Dashboards rely mainly on traditional system logs. Physical sensors are used in only a few examples to automate acquisition of audio, video or other relevant data about the learner or her environment. In addition, our work is one of the few examples where manual tracking tools are integrated in the analytics process. The choice of sensor types has big consequences with respect to completeness and usefulness of data. In our work, students consider incomplete data about learning activities a major bottleneck that hinders adoption and engagement with the use of dashboard applications. Data completeness depends on the types of trackers as well as on how the tracked software is used in the course. Thus, a thorough assessment of data completeness of different sensors is important to build dashboards that are useful for learners.

3 The learning setting has an influence on the potential impact of learning dashboards. In our work, we received better evaluation results in settings where StepUp! was deployed to support students working in groups and sharing a topic than students working individually on different topics [35]. Further research is needed to identify the settings in which these approaches work well, as well as to identify shortcomings and solutions for settings in which dashboards have not been deployed successfully.

4 Evaluation of learning analytics dashboards is often complex. Although many researchers have conducted usefulness and usability evaluations by asking teachers or students to perform a set of tasks, little is known about the usefulness of learning analytics dashboards to solve real issues and needs of students or teachers. Asking students about their actual issues can help to better understand their needs, and is key to better target dashboards towards 
improvements in effectiveness of learning [35]. Furthermore, as perceptions are not always accurate, actual use analysis and other evaluation techniques need to be researched to complement analysis of student perceptions. Such a combined approach is needed to elaborate more solid evaluation methodologies that can be used to assess the impact of learning analytics dashboards.

5 There are also additional benefits that this kind of research can deliver, such as the dataTEL initiative [56] that aims to evolve 'learning science' into more of a data driven science from the collection of beliefs and assumptions that characterizes much of it now. One of the challenges that dataTEL tries to address is the collection and sharing of datasets and evaluation results to enable comparative evaluation studies. A key requirement to better understand potential impact of learning dashboards is to elaborate common evaluation methodologies as well as to share research data among researchers to compare and contrast the usefulness of dashboards in different learning settings.

Moreover, there are other concerns that may impact on how such tools are received, like privacy (even though the sensibilities in this area evolve with the technology [54]) and concerns about when support becomes coercive [55], or questions around technical architecture and interoperability. With the SOLAR society (http://www.solarresearch.org) and the LAK conference series [42], we intent to continue working on these issues.

We hope that this paper can clarify some of the HCI issues involved and can help shape future research in this exciting area.

\section{Acknowledgments}

We thank all evaluation participants for their most valuable feedback. The research leading to these results has received funding from the European Community Seventh Framework Programme (FP7/2007-2013) under grant agreement no. 231396 (ROLE). Katrien Verbert is a post-doctoral fellow of the Research Foundation - Flanders (FWO).

\section{References}

1. Chatti MA, Agustiawan MR, Jarke M, Specht M (2012) Toward a Personal Learning Environment Framework. In: Design, Implementation, and Evaluation of Virtual Learning Environments. IGI Global, 2012. 20-40. Web. 14 Dec. 2012. doi:10.4018/9781-4666-1770-4.ch003 
2. Duval E, Wiley D (2010) Guest Editorial : Open Educational Resources. In: IEEE Trans. on Learning Technologies, 3(2):83-84.

3. Purpura S, Schwanda V, Williams K, Stubler W, Sengers P (2011) Fit4life: the design of a persuasive technology promoting healthy behavior and ideal weight. In: Proceedings of the SIGCHI Conference on Human Factors in Computing Systems (CHI '11), (pp. 423432), ACM, New York, NY, USA.

4. Duval E (2011) Attention please! Learning analytics for visualization and recommendation. In: Proceedings of LAK11, (pp. 9-17), ACM, New York, USA

5. Pechenizkiy M, et al., (eds.) Proceedings of EDM11: 4th International Conference on Educational Data Mining, 2011.

6. Santos JL, Govaerts S, Verbert K, Duval E (2012) Goal-oriented visualizations of activity tracking: a case study with engineering students. In: Shum SB, Gasevic D, Ferguson R (Eds) Proceedings of the 2nd International Conference on Learning Analytics and Knowledge (LAK '12), (pp. 143-152), ACM, New York, NY, USA.

7. Verbert K, Duval E, Klerkx J, Govaerts S, Santos JL (2013) Learning Analytics Dashboard Applications. American Behavioral Scientist, 10 pages. Published online February 2013.

8. Yu Y-C, You S-CD, Tsai D-R (2012) Social interaction feedback system for the smart classroom. In: Proceedings of the 2012 IEEE International Conference on Consumer Electronics (ICCE), (pp. 500-501), IEEE.

9. Pohl A, Bry F, Schwarz J, Gottstein M (2012) Sensing the Classroom: Improving Awareness and Self-Awareness of Students with Backstage. In: Proceedings of the International Conference on Interactive and Collaborative Learning (ICL), Villach, Austria (26-28 September 2012)

10. Barr J, Gunawardena A (2012). Classroom salon: a tool for social collaboration. In Proceedings of the 43rd ACM technical symposium on Computer Science Education (SIGCSE '12), (pp. 197-202), ACM, New York, NY, USA.

11. Fagen W, Kamin S (2012) Developing Device-independent Applications for Active and Collaborative Learning with the SLICE Framework. In Amiel T, Wilson B (Eds), Proceedings of World Conference on Educational Multimedia, Hypermedia and Telecommunications 2012 (pp. 1565-1572). Chesapeake, VA: AACE.

12. Cuendet S, Bonnard Q, Kaplan F, Dillenbourg P (2011) Paper interface design for classroom orchestration. In CHI '11 Extended Abstracts on Human Factors in Computing Systems (CHI EA '11). (pp. 1993-1998), ACM, New York, NY, USA. 
13. Son LH (2012) Supporting Reflection and Classroom Orchestration with Tangible Tabletops. PhD Thesis http://biblion.epfl.ch/EPFL/theses/2012/5313/5313_abs.pdf

14. Martinez Maldonado R, Kay J, Yacef K, Schwendimann B (2012) An interactive teacher's dashboard for monitoring groups in a multi-tabletop learning environment. In: Cerri et al. (Eds) Proceedings of the 11th international conference on Intelligent Tutoring, (pp. 482-492), Springer-Verlag, Berlin, Heidelberg.

15. Gutiérrez Rojas I, Crespo García RM, Delgado Kloos C (2011) Orchestration and feedback in lab sessions: improvements in quick feedback provision. In Delgado Kloos C et al. (Eds) ECTEL 2011 Towards Ubiquitous Learning 6th European Conference of Technology Enhanced Learning 2023 September 2011 Palermo Italy Proceedings LNCS 6964 (Vol. 6964, pp. 424-429). Springer Berlin Heidelberg.

16. Gutiérrez Rojas I, Crespo García RM (2012) Towards Efficient Provision of Feedback Supported by Learning Analytics. In: Proceedings of the 12th International Conference on Advanced Learning Technologies (ICALT), (pp. 599-603), IEEE

17. Morris MR, Piper AM, Cassanego T, Winograd T (2005) Supporting Cooperative Language Learning: Issues in Interface Design for an Interactive Table. Stanford University Technical Report, 2005.

18. Arnold KE, Pistilli MD (2012) Course signals at Purdue: using learning analytics to increase student success. In Shum SB, Gasevic D, Ferguson R (Eds) Proceedings of the 2nd International Conference on Learning Analytics and Knowledge (LAK '12) (pp. 267270). NY: ACM.

19. Dollár A, Steif PS (2012) Web-based Statics Course with Learning Dashboard for Instructors. In Uskov V (Ed) Proceedings of Computers and Advanced Technology in Education (CATE 2012), June 25 - 27, 2012, Napoli, Italy.

20. Govaerts S, Verbert K, Duval E, Pardo A (2012) The student activity meter for awareness and self-reflection, The ACM SIGCHI Conference on Human Factors in Computing Systems, Austin, TX, USA, 5-10 May 2012, CHI EA '12: Proceedings of the 2012 ACM Annual Conference Extended Abstracts on Human Factors in Computing Systems Extended Abstracts, (pp. 869-884), ACM.

21. Ali L, Hatala M, Gašević D, Jovanović J (2012). A qualitative evaluation of evolution of a learning analytics tool. Computers and Education, 58(1):470-489.

22. Podgorelec V, Kuhar S (2011) Taking Advantage of Education Data: Advanced Data Analysis and Reporting in Virtual Learning Environments. Electronics and Electrical Engineering, 114(8):111-116.

23. Leony D, Pardo A, de la Fuente Valentín L, Sánchez de Castro D, Delgado Kloos C (2012) GLASS: a learning analytics visualization tool. In Buckingham Shum S, Gasevic 
D, Ferguson R (Eds) Proceedings of the 2nd International Conference on Learning Analytics and Knowledge LAK '12, (pp. 162-163). ACM, New York, NY, USA.

24. Scheuer O, Zinn C (2007) How did the e-learning session go? The Student Inspector. In: Luckin R, et al. (Eds) Proceedings of the 2007 conference on Artificial Intelligence in Education: Building Technology Rich Learning Contexts That Work, (pp. 487-494), IOS Press, Amsterdam, The Netherlands.

25. Lafford BA (2004) Review of Tell Me More Spanish, Journal on Language Learning \& Technology, 8(3):21-34.

26. Kerly A, Ellis R, Bull S (2007) CALMsystem: A Conversational Agent for Learner Modelling. In: Ellis R, et al. (Eds), Applications and Innovations in Intelligent Systems XV - Proceedings of AI-2007, 27th SGAI International Conference on Innovative Techniques and Applications of Artificial Intelligence (pp. 89-102). Springer Verlag.

27. Chen GD, Chang CK, Wang CY (2008) Ubiquitous learning website: Scaffold learners by mobile devices with information-aware techniques, Computers \& Education, 50(1): $77-90$.

28. Kobsa E, Dimitrova V, Boyle R (2005) Using student and group models to support teachers in web-based distance education. Proc. of the 10th international conference on user modeling (pp. 124-133). Edinburgh, UK.

29. Santos Odriozola J, Verbert K, Duval E (2012) Empowering students to reflect on their activity with StepUp!: two case studies with engineering students. In: Proceedings of ARTELL'12 2nd workshop on Awareness and Reflection, (pp. 73-86), CEUR Workshop Proceedings.

30. Nakahara J, Hisamatsu S, Yaegashi K, Yamauchi Y (2005) iTree: does the mobile phone encourage learners to be more involved in collaborative learning? In: Proceedings of th 2005 conference on Computer support for collaborative learning: learning 2005: the next 10 years! (CSCL '05). International Society of the Learning Sciences, (pp. 470-478).

31. Dawson S, Bakharia A, Heathcote E (2010) SNAPP: Realising the affordances of realtime SNA within networked learning environments. In: Dirckinck- Holmfeld L, et al. (Eds), Proceedings of the 7th International Conference on Networked Learning (pp. 125133). Lancaster: University of Lancaster.

32. Silius K, Miilumaki T, Huhtamaki J, Tebest T, Merilainen J, Pohjolainen S (2010) Students' Motivations for Social Media Enhanced Studying and Learning. Knowledge Management \& E-Learning: An International Journal (KM\&EL), 2(1):51-67.

33. France L, Heraud J-M, Marty J-C, Carron T, Heili J (2006) Monitoring Virtual Classroom: Visualization Techniques to Observe Student Activities in an e-Learning 
System. In: Proceedings of the Sixth International Conference on Advanced Learning Technologies, (pp.716-720), IEEE.

34. Govaerts S, Verbert K, Dahrendorf D, Ullrich C, Schmidt M, Werkle M, Chatterjee A, Nussbaumer A, Renzel D, Scheffel M, Friedrich M, Santos Odriozola J, Duval E, Law E (2011) Towards Responsive Open Learning Environments: the ROLE Interoperability framework. In Proceedings of 6th European Conference of Technology Enhanced Learning, EC-TEL 2011, LNCS Vol. 6964. (pp. 125-138) Springer.

35. Santos JL, Verbert K, Govaerts S, Duval E (in press) Addressing learner issues with StepUp!: an Evaluation. In Suthers D, Verbert K, Duval E, Ochoa X (Eds) Proceedings of the 2013 ACM International Conference on Learning Analytics and Knowledge (LAK'13), 10 pages. ACM.

36. Verbert K, Manouselis N, Ochoa X, Wolpers M, Drachsler H, Bosnic I, Duval E (2012) Context-aware Recommender Systems for Learning: a Survey and Future Challenges. IEEE Transactions on Learning Technology, 5(4):318-335.

37. Verpoorten D, Westera W, Specht M (2011) Reflection amplifiers in online courses: a classification framework. Journal of Interactive Learning Research, 22(2):167-190.

38. Leony D, Crespo RM, Perez-Sanagustin M, Parada GHA, de la Fuente Valentin L, Pardo A (2012). Coverage Metrics for Learning- Event Datasets Based on Client-Side Monitoring. Proceedings of the IEEE 12th International Conference on Advanced Learning Technologies (ICALT), 2012 (pp. 652-653), IEEE.

39. Dillenbourg P, Zufferey G, Alavi H, Jermann P, Do-lenh S, Bonnard Q, Cuendet S, et al. (2011) Classroom Orchestration: The Third Circle of Usability. CSCL2011 Proceedings (Vol. I, pp. 510-517), Hong Kong: International Society of the Learning Sciences.

40. Bakker J, Holenderski L, Kocielnik R, Pechenizkiy M, Sidorova N (2012) Stess@Work: from measuring stress to its understanding, prediction and handling with personalized coaching. In Proceedings of the 2nd ACM SIGHIT International Health Informatics Symposium (IHI '12), (pp. 673-678), ACM, New York, NY, USA.

41. Shum SB, Gasevic D, Ferguson R (Eds) (2012) LAK12: Proceedings of 2nd International Conference on Learning Analytics and Knowledge. ACM.

42. Suthers D, Verbert K, Duval E, Ochoa X (Eds) (2013) LAK13: Proceedings of 3rd International Conference on Learning Analytics and Knowledge. ACM.

43. Schaaff K, Müller L, Kirst M, Heuer S (2012) xAffect - A Modular Framework for Online Affect Recognition and Biofeedback Applications. Proceedings of the 3rd International Workshop on Motivational and Affective Aspects of Technology Enhanced Learning, September 2012

44. Leony D, Pardo A, Parada Gélvez H, Delgado Kloos C (2012). A widget to recommend learning resources based on the learner affective state. Proceedings of the $3 \mathrm{rd}$ 
International Workshop on Motivational and Affective Aspects of Technology Enhanced Learning, September 2012

45. Harris S (2010) The Place of Virtual, Pedagogic and Physical Space in the 21st Century Classroom. 2010

46. Bizer C (2009) The Emerging Web of Linked Data. IEEE Intelligent Systems, 24(5):8792

47. Siemens G, Gasevic D, Haythornthwaite C, Dawson S, Buckingham Shum S, Ferguson R, Duval E, Verbert K, Baker RSJd (2011) Open Learning Analytics : an integrated \& modularized platform Proposal to design, implement and evaluate an open platform to integrate heterogeneous learning analytics techniques. Retrieved online from http://solaresearch.org/OpenLearningAnalytics.pdf

48. Baillie, C (2000) Motivation and attrition in engineering students, Geraldine Fitzgerald European Journal of Engineering Education, 25(2):145-155.

49. Killen R (1994) Differences between students' and lecturers' perceptions of factors influencing students' academic success at university. Higher Education Research and Development, 13, 199. 1994.

50. Zhou X, Jin Q (2011) Dynamical user networking and proling based on activity streams for enhanced social learning. Advances in Web-Based Learning-ICWL (pp. 219-225), Springer.

51. Goligoski E (2012) Motivating the learner: Mozillas open badges program. Access to Knowledge: A Course Journal 4(1), 8 pages.

52. Domínguez A, Saenz-de-Navarrete J, de-Marcos L, Fernández-Sanz L, Pagés C, Martínez-Herráiz J-J (2013) Gamifying learning experiences: Practical implications and outcomes, Computers \& Education, 63:380-392, April 2013, Elsevier.

53. Santos JL, Charleer S, Parra G, Klerkx J, Duval E and Verbert K (to appear) Motivating Students in an Open Learning Environment through Open Badges. Proceedings of the Eighth European Conference on Technology Enhanced Learning (ECTEL 2013), 14 pages (to appear).

54. Jarvis J (2011) Public Parts: How Sharing in the Digital Age Improves the Way We Work and Live. Simon \& Schuster.

55. Purpura S, Schwanda V, Williams K, Stubler W, Sengers P (2011) Fit4life: the design of a persuasive technology promoting healthy behavior and ideal weight. Proceedings of the 2011 annual conference on Human factors in computing systems (pp. 423-432). ACM.

56. Verbert K, Manouselis N, Drachsler H, Duval E (2012) Dataset-driven research to support learning and knowledge analytics. Educational Technology and Society, 15(3):133-148. 
[PUC EIST Special Issue Template]

DOI XX.XXXX/sXXXXX-XXX-XXXX-X 